\title{
QUANTIFICAÇÃO DE MULLITA PROVENIENTE DE RESÍDUOS DE CAULIM DA REGIÃO AMAZÔNICA: USO DO MÉTODO DE RIETVELD
}

\author{
Marlice C. Martellia, Eric Mochiuttia,*,(i), João Pedro O. Lima ${ }^{\text {a }}$ e Roberto de F. Neves ${ }^{\mathrm{a}}$ \\ ${ }^{a}$ Faculdade de Engenharia Química, Universidade Federal do Pará, 66075-110 Belém - PA, Brasil
}

Recebido em 07/08/2020; aceito em 17/11/2020; publicado na web em 22/12/2020

\begin{abstract}
QUANTIFICATION OF MULLITE FROM KAOLIN WASTES FROM THE AMAZON REGION: USE OF THE RIETVELD METHOD. Mullite is used to obtain a refractory material, there are several factors that influence the synthesis process of mullite: the preparation of the mixture, the precipitation and the reaction of $\mathrm{SiO}_{2}$ and $\mathrm{Al}_{2} \mathrm{O}_{3}$. For the synthesis of mullite, samples of kaolin processing residues were used as precursor material, because it presents $\mathrm{SiO}_{2}$ and $\mathrm{Al}_{2} \mathrm{O}_{3}$ in its composition. This work aimed to identify, by X-ray diffraction, and quantify the mineral phases present in samples of kaolin processing residues from the Amazon region calcined at 1300,1400 and $1500{ }^{\circ} \mathrm{C}$, using the Rietveld method. The method allowed the refinement of the complex crystalline structures and was applied to the data supply for quantitative analyses with satisfactory results of good accuracy. The results of the quantification of crystalline and non-crystalline phases (with internal standard) in the samples calcined at $1500{ }^{\circ} \mathrm{C}$ presented approximate values of mullite (62\%), cristobalite (32\%) and non-crystalline phases (6\%), for both samples, indicating that the refinement model applied is optimal. These results obtained from the quantification of the phases by the method of Rietveld are presenting coherent and satisfactory values, in comparison with the theoretical ones by the phase diagram $\mathrm{Al}_{2} \mathrm{O}_{3}$ and $\mathrm{SiO}_{2}$.
\end{abstract}

Keywords: X-ray diffraction; Kaolin; Rietveld; Mullite.

\section{INTRODUÇÃO}

A região amazônica dispõe de depósitos de caulim que são adequados a cobertura de papel. No estado do Pará, existem três indústrias de beneficiamento de caulim para papel. Estas indústrias geram mais de 1 milhão de toneladas de resíduo por ano. ${ }^{1}$ Os resultados da composição indicam que o resíduo é formado, principalmente, por caulinita, ${ }^{2}$ sendo que, a sua granulometria é inadequada para a produção de papel, tornando esse material aplicável em outros processos como matéria-prima. ${ }^{3} \mathrm{Uma}$ das diversas aplicações é como matéria-prima para a produção de refratários sílico-aluminosos, como os mulíticos. ${ }^{4}$

Para a formação da mullita sinterizada, de fórmula $\mathrm{Al}_{6} \mathrm{Si}_{2} \mathrm{O}_{13}$, utilizam-se como materiais de partida óxidos, hidróxidos, sais e silicatos de Al. A temperatura de mulitização é controlada pelo tamanho das partículas dos pós de partida, pelo grau de ordem estrutural da caulinita e pela presença das impurezas, que influenciam na formação da mullita promovendo a cristalização de fases minerais diferentes e aumentando a resistência. ${ }^{5}$

A difração de raios $\mathrm{X}$ em pó é um dos métodos para obtenção de informações de fase quantitativa a partir de misturas multicomponentes. Contudo, as análises de raios X têm sido tradicionalmente dependentes de vários parâmetros, incluindo características da amostra, pureza e disponibilidade de padrões e a qualidade dos dados de difração. ${ }^{6}$

Existem muitos métodos citados na literatura para a análise quantitativa de fases, neste trabalho será apresentado o desenvolvido por Rietveld, ${ }^{7,8}$ um dos mais utilizados para refinamento de parâmetros, tanto dos dados de difração de raios $\mathrm{X}$, quanto dos de difração de nêutrons. ${ }^{6,9},{ }^{10}$

$\mathrm{O}$ método de Rietveld foi projetado originalmente para o refinamento de estruturas cristalinas e magnéticas a partir de dados de neutrões de pó, mas hoje em dia também é possível determinar quantitativamente as quantidades de fases cristalinas mesmo em amostras complexas. ${ }^{6,9}$

\footnotetext{
*e-mail: mochiuttieric@gmail.com
}

Esse método tem por base a simulação do perfil difratométrico, a partir dos dados difratométricos teóricos das fases identificadas em uma amostra, analisando todo o padrão difratométrico para o refinamento e permitindo calcular os parâmetros geométricos e os cristalográficos que melhor se ajustem aos da amostra. Esse ajuste em cada ponto é feito aplicando a técnica dos mínimos quadrados.

Para aplicar o método de Rietveld, é necessário conhecer a estrutura das fases componentes da mistura com um bom grau de aproximação, possuir dados difratométricos de boa qualidade e ter um bom conhecimento em cristalografia. O método permite o refinamento de estruturas cristalinas complexas e é aplicado ao fornecimento de dados para análise quantitativa com resultados satisfatórios de boa precisão. Este trabalho teve como objetivo identificar as fases minerais, por difração de raios $\mathrm{X}$, de resíduos de beneficiamento de caulim calcinados a 1300,1400 e $1500{ }^{\circ} \mathrm{C}$, aplicar o método de Rietveld, por difração de raios X, para a quantificação de fases presentes em amostras de resíduos de beneficiamento de caulim da região Amazônica e, por fim, comparar os resultados entre os dois resíduos estudados, avaliando a precisão do método aplicado.

\section{PARTE EXPERIMENTAL}

Os materiais utilizados no presente trabalho foram resíduos do processo de beneficiamento de caulim da região do rio Capim (CC) e do rio Jari $(\mathrm{CJ})$, do estado do Pará. A Figura 1 apresenta o mapa geográfico da região onde esses rios se encontram, destacando a localização de ambas as minas onde as amostras foram coletadas.

\section{Formação de mullita}

Baseando-se em diagramas de fases $\mathrm{Al}_{2} \mathrm{O}_{3}-\mathrm{SiO}_{2},{ }^{11-13}$ encontrados na literatura, são observados os produtos ou fases formadas com a variação de temperatura $\left({ }^{\circ} \mathrm{C}\right)$ em função da composição molar ou de massa dos componentes. Através desse diagrama, pode-se determinar o valor teórico máximo previsto de formação de mullita e de cristobalita em relação ao material de partida. Para uma argila 


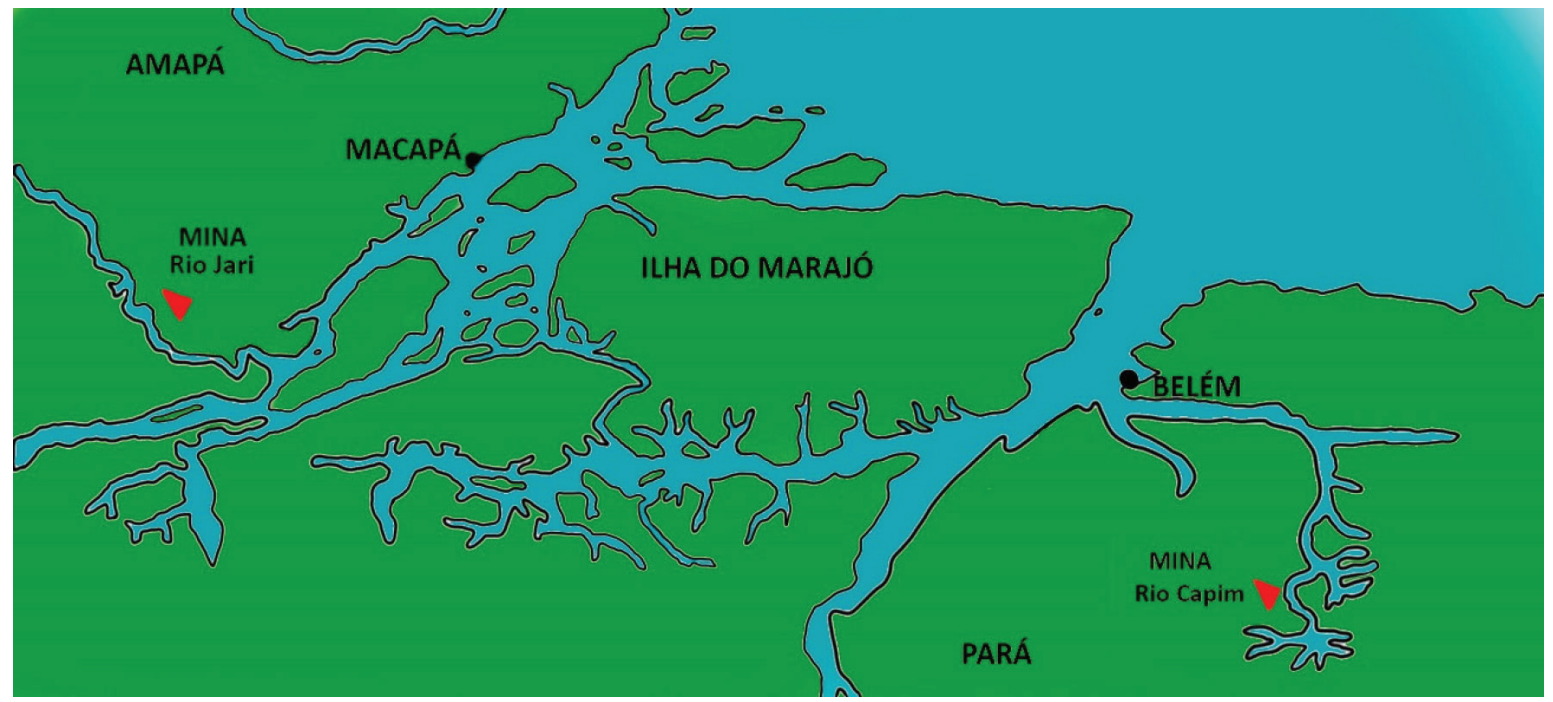

Figura 1. Mapa geográfico da região dos rios Capim e Jari

essencialmente caulinítica, esses valores podem ser determinados partindo-se da composição em massa da metacaulinita. Através do processo de tratamento térmico da caulinita, com temperaturas acima de $540{ }^{\circ} \mathrm{C}$, ocorre a desidroxilação dessa estrutura, caracterizando assim a formação de metacaulinita, em que a caulinita tem sua estrutura modificada de uma conformação octaédrica de $\mathrm{Al}^{3+}$ para tetraédrica. ${ }^{2,4}$ A metacaulinita corresponde a uma transformação de fase não cristalina da caulinita, tendo como composição $\mathrm{Al}_{2} \mathrm{O}_{3}$ (46\%) e de $\mathrm{SiO}_{2}$ (54\%), obtendo-se assim: $64 \%$ de mullita e $36 \%$ de cristobalita. ${ }^{11-13}$

\section{Análise química}

As análises de composição química foram conduzidas para as amostras de rejeitos $\mathrm{CJ}$ e $\mathrm{CC}$ originais e essas calcinadas a $1000^{\circ} \mathrm{C}$. As técnicas de determinação dos teores dos óxidos foram: $\mathrm{SiO}_{2}$ por gravimetria (método clássico); $\mathrm{Al}_{2} \mathrm{O}_{3}$ por titrimetria (método clássico); $\mathrm{Fe}_{2} \mathrm{O}_{3}$ e $\mathrm{TiO}_{2}$ por espectrofotometria (método colorimétrico); $\mathrm{Na}_{2} \mathrm{O}, \mathrm{K}_{2} \mathrm{O}, \mathrm{CaO}$ e $\mathrm{MgO}$ por espectrometria de absorção atômica. A calcinação foi realizada em forno mufla por 3 horas, a uma velocidade de aquecimento de $10{ }^{\circ} \mathrm{C} / \mathrm{min}$, em presença de ar, para garantir a eliminação de todo o material orgânico presente. Através da calcinação a $1000{ }^{\circ} \mathrm{C}$, determinou-se a perda de massa ao fogo dos rejeitos.

Para identificação das amostras de resíduos do beneficiamento de caulim, foram adicionados os índices de 1300, 1400 e 1500 referentes às temperaturas de calcinação $\left({ }^{\circ} \mathrm{C}\right)$ a que foram submetidas.

\section{Difração de raios $X$}

Para a identificação mineralógica, determinação do grau de ordem/desordem das caulinitas e quantificação das fases cristalinas dos materiais calcinados a temperaturas elevadas, foi utilizado um aparelho de difração de raios X com controle automático, marca PHILIPS, modelo PW 3710 acoplado a um microcomputador. Goniômetro PHILIPS, modelo PW 3020, com fenda automática e monocromador de grafite, tubo de raios $\mathrm{X}$ com ânodo de cobre, operando a $45 \mathrm{kV}$ e $40 \mathrm{~mA}$. A varredura foi contínua de $5^{\circ}$ a $65^{\circ} 2 \theta$, empregando-se o método do pó, em lâmina escavada de vidro, com preenchimento back loading, contagem de $0,4 \mathrm{~s}$, com passo de 0,02 $\left({ }^{\circ} 2 \theta\right)$. Essas análises foram realizadas no Laboratório de difração de raios X no CG/UFPA. Os difratogramas foram interpretados com os softwares APD (Automated Powder Diffraction) da PHILIPS e com as fichas PDF (Powder Diffraction File) do ICDD (International Center for Diffraction Data).

\section{Método de Rietveld}

Existem alguns programas que executam o método de Rietveld, dois deles foram estudados, o RIQAS e o FULLPROF, ambos encontrados e disponíveis em veículo eletrônico de comunicação, sendo o último com mais parâmetros de refinamento que o primeiro, portanto, o resultado deste é o apresentado.

Para iniciar as etapas de refinamento, foi utilizado um difratograma mais representativo da amostra, e como essa se apresenta composta por muitas fases, modelos estruturais foram inseridos para cada fase identificada. Os primeiros ajustes foram os de fator de escala e fator de fundo, para ajustar a linha base (background), os parâmetros da célula unitária de cada fase foram refinados para corrigir o deslocamento dos picos do difratograma, os parâmetros de perfil (largura, forma e simetria) para o ajuste da altura e do perfil geral dos picos. Posteriormente, outros parâmetros foram refinados e, em cada etapa do refinamento, vários ciclos executados.

A qualidade do refinamento foi verificada através de indicadores estatísticos numéricos, durante os cálculos e após o término desses, indicando se o refinamento estava sendo bem processado. No FullProf, dois destes índices são os usados: O fator $\mathrm{R}_{\text {Bragg, }}$, Equação $1^{14}$ que é utilizado na indicação dos modelos para cada fase e determina o desvio entre o perfil do difratograma observado e o calculado. Isso quer dizer que o ajuste perfeito ocorre quando este fator é zero. É considerado um ótimo refinamento quando esse valor é em torno de três e um refinamento aceitável quando está em torno de nove, por ser assim o usual na prática. ${ }^{15} \mathrm{O}$ outro é o índice Goodness Off Fit (GOF ou S), apresentado por amostra, correlaciona tanto parâmetros estruturais quanto de perfil e é calculado pela relação entre o fator Rwp (residual ponderado calculado), Equação $2^{14}$ e o fator Re (residual esperado), Equação 3. ${ }^{14}$ Valores típicos de Rwp para refinamentos utilizando difração de raios X variam de $10 \%$ a $20 \% .{ }^{15}$

$\mathrm{O}$ valor de GOF, apresenta o melhor refinamento quando atinge o valor unitário, porém, na literatura, ${ }^{14,15}$ é citado que valores mais elevados desse índice podem ser aceitáveis, visto que podem ocorrer a presença de fase não cristalina e fases minoritárias. Como citado por esses autores e verificado também neste trabalho, o tratamento das fases minoritárias é uma das dificuldades do método de Rietveld, pois, como apresenta uma concentração muito baixa, os picos no difratograma confundem com a radiação de fundo. Portanto, no intuito 
de alcançar um melhor ajuste, essas fases não foram consideradas para o refinamento.

$$
R_{\text {Bragg }}=100 \frac{\sum_{k}\left|I_{k}(o b s)-I_{k}(c a l c)\right|}{\sum_{k}\left|I_{k}(o b s)\right|}
$$

Em que $I_{k}(o b s)$ e $I_{k}($ calc $)$ são as intensidades integrada observada e calculada da reflexão com índices hkl, contidas no índice $\mathrm{k}$, respectivamente. $\mathrm{O}$ índice $\mathrm{k}$ são os índices de Miller, h, k, 1 que contribuem na intensidade.

$$
R_{w p}=100\left[\frac{\sum_{i} w_{i} \mid y_{i}(o b s)-\left.y_{i}(\text { calc })\right|^{2}}{\sum_{i} w_{i}\left|y_{i}(o b s)\right|^{2}}\right]^{1 / 2}
$$

sendo que i corresponde a cada ponto medido; $y_{i}($ obs $)$ e $y_{i}($ calc $)$ são as intensidades observadas e as calculadas na posição $2 \theta_{\mathrm{i}}$, respectivamente e, $w_{i}$ é o peso estatístico observado na posição $2 \theta_{i}$.

$$
R_{e}=100\left[\frac{N-P+C}{\sum_{i} w_{i} y_{i}^{2}(o b s)}\right]^{1 / 2}
$$

Em que $(N-P+C)$ é o número de graus de liberdade ( $\mathrm{N}$ é o número de pontos do difratograma, $\mathrm{P}$ é o número de parâmetros refinados e C é o número de equações de vínculo entre os parâmetros).

Em cerâmica é conhecido que o material não cristalino, como sílica vítrea, influencia nas propriedades termoquímicas dos produtos. A técnica utilizada neste trabalho para determinar a quantidade de fase não cristalina foi a do padrão interno, ${ }^{6}$ que, no refinamento por Rietveld, requer a inclusão de um padrão com estrutura e concentração conhecidas.

Nesse caso, foram utilizados $20 \%$ em peso de fluorita, como padrão interno, adicionado a $80 \%$ em peso de rejeito do caulim CC-1500, amostra original. Depois de determinar todas as fases cristalinas presentes na amostra, foram obtidas as frações em peso de cada fase, incluindo a quantidade do padrão interno adicionado. A soma de todas as frações deve ser igual a 100\%, se não atingir esse valor, a diferença é o teor de material não cristalino.

O fator de normalização, Equação 4, é determinado em relação a quantidade de fluorita adicionada à amostra original.

$$
f_{n}=\frac{W_{f}}{W_{f r}}
$$

sendo $f_{n}, W_{f}$ e $W_{f r}$, respectivamente, o fator de normalização, a fração em peso (\%) de fluorita acrescentada na amostra original (CC-1500) e a fração em peso (\%) de fluorita determinada no refinamento.

Para calcular as frações em peso (\%) de cada fase normalizada $\left(W_{m 1}\right.$ e $\left.W_{c 1}\right)$ na mistura sintética CC80F20, utiliza-se as Equação 5 e 6.

$$
\begin{aligned}
& W_{m 1}=W_{m r 1} \cdot f_{n} \\
& W_{c 1}=W_{c r 1} \cdot f_{n}
\end{aligned}
$$

sendo $W_{m 1}, W_{m r 1}, W_{c 1}$ e $W_{c r 1}$ representam, respectivamente, a fração de peso (\%) da mullita, a fração em peso (\%) da mullita determinada através do refinamento, a fração em peso (\%) de cristobalita e a fração em peso $(\%)$ de cristobalita determinada através do refinamento. Todos estes para a mistura sintética CC80F20.

A Equação 7, demonstra a forma de cálculo da fração em peso $(\%)$ da fase não cristalina da mistura sintética $\left(W_{\text {não-cristalina }}\right)$.

$$
W_{\text {não-cristalina }}=100-\left(W_{m 1}+W_{c 1}+W_{f 1}\right)
$$

Outra maneira de determinar $W_{\text {não-cristalin }}$ na mistura sintética é utilizando o fator de normalização $\left(f_{n}\right)$ na amostra original CC-1500.

$$
\begin{aligned}
& W_{m 2}=W_{m r 2} \cdot f_{n} \\
& W_{c 2}=W_{c r 2} \cdot f_{n}
\end{aligned}
$$

em que, $W_{m 2}, W_{m r 2}, W_{c 2}$ e $W_{c r 2}$ são, respectivamente, fração de peso (\%) da mullita, a fração em peso (\%) da mullita determinada através do refinamento, a fração em peso $(\%)$ de cristobalita e a fração em peso $(\%)$ de cristobalita determinada através do refinamento, estes valores em função da amostra original CC-1500.

Para determinação da fração em peso (\%) da fase não cristalina, utiliza-se a Equação 10, a seguir.

$$
W_{\text {não-cristalina }}=100-\left(W_{m 2}+W_{c 2}\right)
$$

Para a base de $100 \%$ da amostra original (CC-1500), as Equações 11,12 e 13, representam, respectivamente os cálculos da fração em peso $(\%)$ de mullita $\left(W_{m 100}\right)$, a fração em peso $(\%)$ de cristobalita $\left(W_{c 100}\right.$ e a fração em peso $(\%)$ da fase não cristalina $\left(W_{\text {não-cristalina } 100}\right.$.

$$
\begin{gathered}
W_{m 100}=\frac{W_{m 1}}{0,8} \\
W_{c 100}=\frac{W_{c 1}}{0,8} \\
W_{\text {não-cristalina } 100}=100-\left(W_{m 100}+W_{c 100}\right)
\end{gathered}
$$

Durante as etapas do refinamento, foi realizada análise visual dos gráficos das diferenças entre os difratogramas calculado e o observado, pois esses permitiram identificar o tipo de parâmetro que poderia estar interferindo no ajuste entre os padrões em cada etapa do refinamento. Os gráficos finais de cada refinamento são apresentados por amostra.

\section{Modelos estruturais}

Etapas preliminares foram realizadas, indicando que a fase majoritária nas amostras analisadas por Rietveld é a mullita, e a de maior interesse para a pesquisa. Por esse motivo, maior detalhe foi dado na escolha do modelo estrutural dessa fase.

A mullita pode ser descrita através da seguinte fórmula $\mathrm{Al}_{4+2 x} \mathrm{Si}_{2-2 \mathrm{x}} \mathrm{O}_{10-\mathrm{x}}$, representando " $\mathrm{x}$ " o número de vacâncias (lacunas) do oxigênio na estrutura de uma célula unitária. Pode-se determinar o valor de "m" e de " $x$ " através da Equação 14 e 15, respectivamente, baseando-se na concentração molar de $\mathrm{Al}_{2} \mathrm{O}_{3}{ }^{16-18}$

$$
\begin{gathered}
m \%=144,5 a-1029,5 \\
x=10-6\left[\frac{m+200}{m+100}\right]
\end{gathered}
$$

sendo o valor de "a" referente ao parâmetro da célula unitária da estrutura em questão. Através desse cálculo, pode-se determinar a estequiometria da estrutura da mullita. Tendo em vista que os valores de " $m$ " e " $x$ " podem variar de $60,0 \%$ até $66,7 \%$ e 0,25 até 0,40 , respectivamente. ${ }^{18}$ Onde, para a conformação $3: 2$ de $\mathrm{Al}_{2} \mathrm{O}_{3} / \mathrm{SiO}_{2}$, temse os parâmetros " $m$ " e "x" iguais à $60,0 \%$ e 0,25 , respectivamente. Para a proporção de 2:1 tem-se os valores desses parâmetros ("m" e " $\mathrm{x}$ ") iguais a $66,7 \%$ e 0,40 .

Então, para o refinamento, quatro modelos de estrutura para a fase mullita encontrados em literatura foram utilizados. ${ }^{19}$ Dois modelos de estrutura de mullita na proporção $2: 1$ e dois com estrutura 3:2. Nos 
primeiros modelos não foram possíveis ajustes satisfatórios, indicando, assim, que a amostra não teria esse padrão de estrutura. Nos modelos 3:2, da mullita $\left(3 \mathrm{Al}_{2} \mathrm{O}_{3} \cdot 2 \mathrm{SiO}_{2}\right),{ }^{5}$ os ajustes foram muito bons, indicando que a amostra apresenta esse tipo de proporção na estrutura.

\section{Microscopia Eletrônica de Varredura (MEV)}

Para as análises de microscopia eletrônica de varredura, utilizouse um microscópio eletrônico de varredura LEO modelo 1450VP. As amostras de $\mathrm{CJ}$ e $\mathrm{CC}$ calcinadas a $1500{ }^{\circ} \mathrm{C}$ foram metalizadas por Au, utilizando-se um metalizador EMITECH K550. A metalização, nesse equipamento, foi feita a partir da interação entre um alvo de Au puro e íons de $\operatorname{Ar}$ (gás argônio), a uma pressão de $2.10^{-1} \mathrm{mbar}$, e corrente de $25 \mathrm{~mA}$ durante $02^{\prime} 30^{\prime \prime}$, resultando na deposição de uma película com espessura média de $\pm 15 \mathrm{~nm}$ (nanômetros) sobre as amostras. As amostras foram montadas em suportes de alumínio com $10 \mathrm{~mm}$ de diâmetro através de fita adesiva de carbono. As imagens foram geradas por detecção de elétrons secundários, utilizando-se aceleração de voltagem de $20 \mathrm{kV}$.

\section{RESULTADOS E DISCUSSÃO}

\section{Análise química}

Os resultados da análise química e da perda de massa ao fogo, de amostras de rejeitos CJ, CC estão apresentados na Tabela 1. Observa-se que os valores da perda de massa para as primeiras amostras de rejeitos e dos teores de alumina e sílica são bastante próximos aos teóricos da caulinita. A composição teórica da caulinita é de: $\mathrm{Al}_{2} \mathrm{Si}_{2} \mathrm{O}_{5}(\mathrm{OH})_{4}\left(39,50 \% \mathrm{Al}_{2} \mathrm{O}_{3}, 46,54 \% \mathrm{SiO}_{2}, 13,96 \% \mathrm{H}_{2} \mathrm{O}\right.$ ou de perda ao fogo). Essa composição é aproximada, variando com o processo de formação da caulinita.

Os teores de alumina apresentados para ambos os rejeitos de beneficiamento de caulim são promissores para a produção de chamota branca com finalidade de aplicação em cerâmica branca. Nos teores de $\mathrm{SiO}_{2}$ total, para CJ e CC, estão incluídos os da caulinita e os de quartzo também presente em pouca quantidade.

Os teores de impurezas mais elevados são os dos óxidos de $\mathrm{Fe}$ e Ti nas amostras de rejeitos. Cerca de 1,5\% do teor de $\mathrm{Fe}$ na amostra CJ está presente dentro da estrutura da caulinita, ${ }^{20,21}$ então, o restante do teor $(0,46 \%)$ está fora da estrutura. Esse teor de $\mathrm{Fe}$ se dá devido a existência de óxidos de ferro (hematita e goethita) presentes na estrutura da caulinita, com valores de
Tabela 1. Análise química e perda de massa ao fogo de rejeitos de caulim

\begin{tabular}{|c|c|c|c|c|c|}
\hline Dados em \% & & Am & & & Teórico \\
\hline Óxidos & $\mathrm{CJ}$ & CJ-1000 & $\mathrm{CC}$ & CC-1000 & Caulinita \\
\hline $\mathrm{SiO}_{2}$ & 44,10 & 50,80 & 46,30 & 53,30 & 46,54 \\
\hline $\mathrm{Al}_{2} \mathrm{O}_{3}$ & 37,40 & 43,20 & 38,40 & 44,60 & 39,50 \\
\hline $\mathrm{Fe}_{2} \mathrm{O}_{3}$ & 1,96 & 2,40 & 0,69 & 0,76 & --- \\
\hline $\mathrm{TiO}_{2}$ & 2,14 & 2,94 & 0,96 & 1,03 & --- \\
\hline $\mathrm{CaO}$ & 0,01 & 0,01 & --- & $<0,01$ & --- \\
\hline $\mathrm{MgO}$ & --- & --- & --- & --- & --- \\
\hline $\mathrm{Na}_{2} \mathrm{O}$ & --- & --- & --- & --- & --- \\
\hline $\mathrm{K}_{2} \mathrm{O}$ & 0,05 & 0,10 & 0,10 & 0,10 & --- \\
\hline $\mathrm{H}_{2} \mathrm{O}$ & 13,70 & 0,41 & 13,60 & 0,27 & 13,96 \\
\hline Total & 99,36 & 99,86 & 100,05 & 100,06 & 100 \\
\hline
\end{tabular}

solubilidade, de aproximadamente $170 \mathrm{mmol} \mathrm{mol}^{-1} \mathrm{e} 330 \mathrm{mmol} \mathrm{mol}^{-1}$, respectivamente. ${ }^{21}$ Os valores que estão muito abaixo de $1 \%$ são mais difíceis de serem detectados por DRX.

\section{Difração de raios X (DRX)}

As Figuras 2 e 3, apresentam os padrões de DRX das amostras de CJ e CC calcinadas a $1300{ }^{\circ} \mathrm{C}, 1400^{\circ} \mathrm{C}$ e $1500{ }^{\circ} \mathrm{C}$, respectivamente. As duas amostras apresentam padrões semelhantes entre si.

Alguns efeitos da elevação da temperatura nessa faixa são observados para ambos os rejeitos:

1. A transformação do quartzo $\left(>1200^{\circ} \mathrm{C}\right)$ e de parte da sílica não cristalina, proveniente da metacaulinita, em cristobalita.

2. Os picos de rutilo passam a apresentar um decréscimo de suas intensidades à medida que a temperatura aumenta, podendo isso ser atribuído ao aumento de formação de mullita e cristobalita, e não da dissolução do rutilo na fase não cristalina (vidro).

3. Nas amostras de CJ, tanto o teor de Fe que estava originalmente na estrutura da caulinita, quanto o que estaria fora, em temperaturas elevadas (acima de $1300^{\circ} \mathrm{C}$ ), podem estar dissolvidos na fase vítrea, pois não foi detectada alguma fase cristalina de mineral de Fe.

\section{Comparação dos resultados entre os resíduos}

Os resultados da quantificação de cada fase presente nas amostras

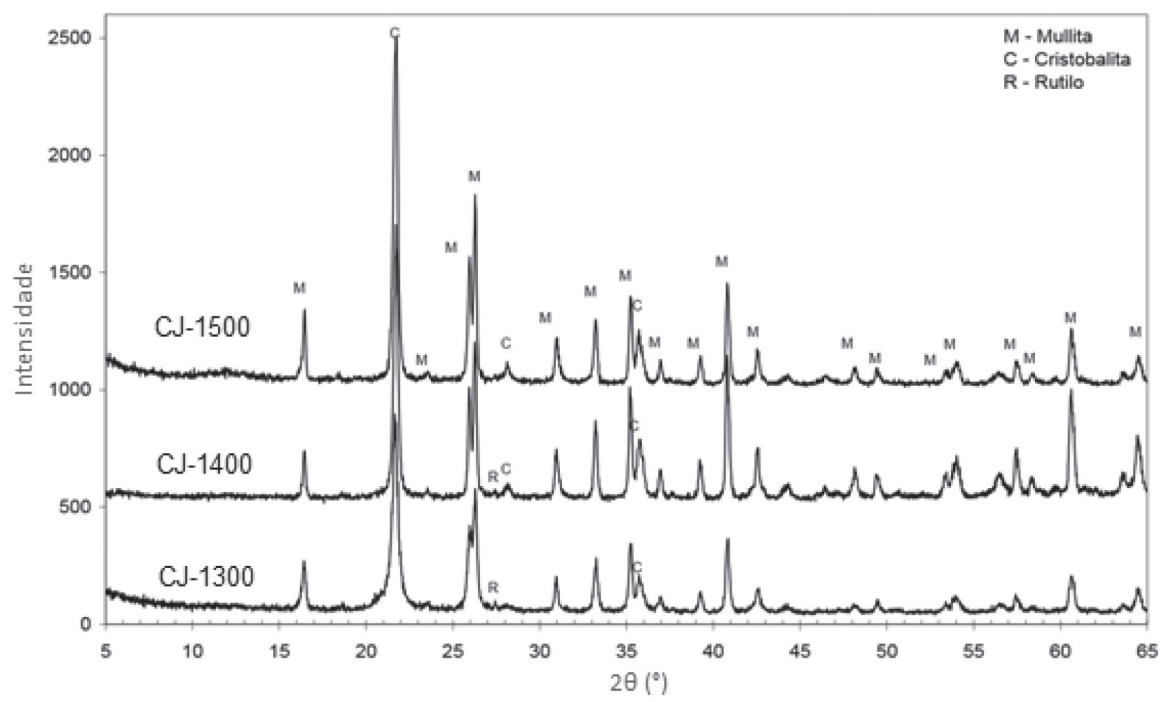

Figura 2. Difratogramas de raios $X$ do rejeito de caulim $\mathrm{CJ}$ calcinado a $1300^{\circ} \mathrm{C}, 1400^{\circ} \mathrm{C}$ e $1500^{\circ} \mathrm{C}$ 


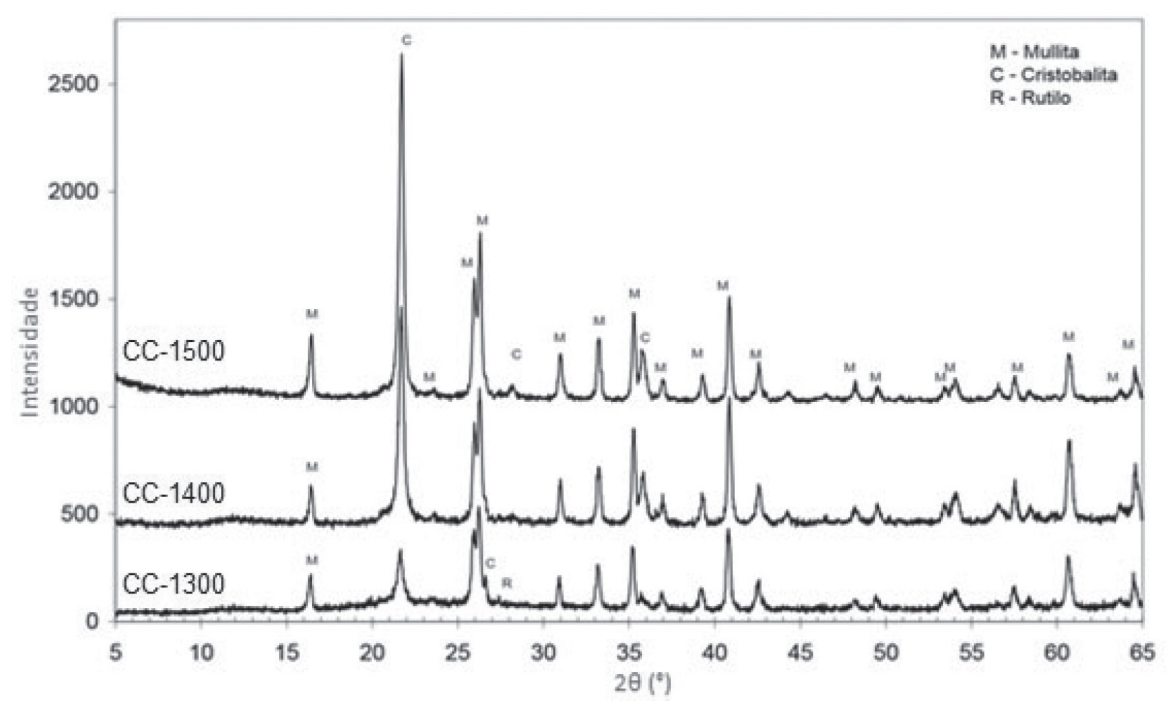

Figura 3. Difratogramas de raios $X$ do rejeito de caulim CC calcinado a $1300^{\circ} \mathrm{C}, 1400^{\circ} \mathrm{C}$ e $1500{ }^{\circ} \mathrm{C}$

dos rejeitos de caulins da região do rio Capim (CC) e do rio Jari (CJ), calcinados nas temperaturas elevadas, são mostrados nas Tabelas 2 e 3, respectivamente. A fase vítrea, presente nas amostras, não foi quantificada, sendo, portanto, uma comparação entre os valores das fases cristalinas, indicativos de refinamento e dos padrões dos difratogramas de cada amostra.

Tabela 2. Quantificação das fases cristalinas pelo refinamento Rietveld, das amostras do rejeito da região do rio Capim, calcinadas em várias temperaturas

\begin{tabular}{lcccccc}
\hline \multirow{2}{*}{ Dados em \% } & \multicolumn{7}{c}{ Amostras } \\
\cline { 2 - 7 } & $\mathrm{CC}-1300$ & $\mathrm{R}_{\text {Bragg }}$ & $\mathrm{CC}-1400$ & $\mathrm{R}_{\text {Bragg }}$ & CC-1500 & $\mathrm{R}_{\text {Bragg }}$ \\
\hline mullita & 81,75 & 8,91 & 64,65 & 4,80 & 65,93 & 2,52 \\
cristobalita & 18,25 & 14,80 & 35,35 & 4,74 & 34,07 & 1,89 \\
\hline Total & 100 & --- & 100 & --- & 100 & --- \\
\hline Rwp & \multicolumn{7}{c}{30,80} & 22,10 & 12,40 \\
\hline
\end{tabular}

Tabela 3. Quantificação das fases cristalinas pelo refinamento Rietveld, das amostras do rejeito da região do rio Jari, calcinadas em várias temperaturas

\begin{tabular}{lcccccc}
\hline \multirow{2}{*}{ Dados em \% } & \multicolumn{7}{c}{ Amostras } \\
\cline { 2 - 7 } & CJ-1300 & $\mathrm{R}_{\text {Bragg }}$ & CJ-1400 & $\mathrm{R}_{\text {Bragg }}$ & CJ-1500 & $\mathrm{R}_{\text {Bragg }}$ \\
\hline mullita & 68,59 & 4,11 & 66,61 & 3,88 & 66,19 & 6,30 \\
cristobalita & 31,41 & 3,63 & 33,39 & 3,54 & 33,81 & 3,95 \\
\hline Total & 100 & --- & 100 & --- & 100 & --- \\
\hline Rwp & \multicolumn{7}{c}{19,50} & 20,50 & 21,50 \\
\hline
\end{tabular}

Através da Tabela 2, verifica-se que a amostra CC-1300 não apresentou um bom refinamento, visto que os indicadores estatísticos estão acima dos aceitáveis. Pelo difratograma dessa amostra se observa que ela apresenta maior quantidade de fase vítrea que as demais, e isto pode ter influenciado para um melhor ajuste dos parâmetros. As amostras de rejeito, CC-1400 e CC-1500, apresentaram valores próximos entre si, o que concorda com as características dos picos dos difratogramas observados, esperando, assim, resultados semelhantes.

Os fatores $\mathrm{R}_{\text {Bragg }}$ dessas últimas estão relativamente baixos, considerados muito bons para análise de Rietveld. Os indicadores residuais Rwp para ambas estão na faixa aceitável, sendo que o da CC-1500 está muito melhor que o da CC-1400.

Os resultados contidos na Tabela 3, das amostras CJ-1300, CJ-1400 e CJ-1500, mostraram que há uma pequena diferença dos teores entre as fases; que os valores de $\mathrm{R}_{\mathrm{Bragg}}$ são considerados baixos e permissíveis para o tipo de refinamento via difração de raios X e que os resultados da quantificação estão coerentes em comparação com os dos padrões de difração.

Deve-se ressaltar que as amostras calcinadas a $1300{ }^{\circ} \mathrm{C}$ e $1400{ }^{\circ} \mathrm{C}$ ainda apresentavam picos minoritários de rutilo, e que pode estar influenciando nos ajustes para a quantificação das fases majoritárias.

De acordo com os resultados da quantificação dos dois rejeitos, $\mathrm{CC}$ e CJ, calcinados a $1500{ }^{\circ} \mathrm{C}$, pode-se observar que apresentaram valores bem próximos entre si.

A Figura 4 apresenta os difratogramas de raios $\mathrm{X}$ dos rejeitos de caulim CJ e CC, calcinados a $1500^{\circ} \mathrm{C}$, em destaque para comparação. Verifica-se que ambas as amostras, CJ-1500 e CC-1500, apresentam os padrões de difração de raios $X$ semelhantes entre si, sendo detectados os picos característicos de mullita e cristobalita.

\section{Refinamento com padrão interno}

Conforme o resultado de DRX, apresentados na Figura 4, as amostras CJ-1500 e CC-1500 foram as que apresentaram apenas as fases mullita e cristobalita e, portanto, uma delas (CC-1500) foi a selecionada para executar o refinamento com padrão interno, pois apresentou melhores valores de $\mathrm{R}_{\mathrm{Bragg}}$ e Rwp.

$\mathrm{Na}$ Tabela 4 são apresentados os valores dos indicadores estatísticos $\mathrm{R}_{\mathrm{Bragg}}$ de cada fase presente e os fatores Rwp das amostras, obtidos no refinamento de Rietveld, através da técnica do padrão interno. As amostras são as do rejeito de caulim da região do rio Capim, calcinadas a $1500{ }^{\circ} \mathrm{C}$, amostra original (CC-1500) e a da mistura de $80 \%$ desse rejeito com $20 \%$ de fluorita, mistura sintética (CC80F20).

Tabela 4. Valores estatísticos do refinamento pelo método de Rietveld

\begin{tabular}{lcc}
\hline \multirow{2}{*}{ Fases das Amostras } & \multicolumn{2}{c}{ Amostras } \\
\cline { 2 - 3 } & $\mathrm{CC} 80 \mathrm{~F} 20$ & $\mathrm{CC}-1500$ \\
\hline $\mathrm{R}_{\text {Bragg }}-$ mullita & 3,82 & 3,76 \\
$\mathrm{R}_{\text {Bragg }}$ - cristobalita & 2,40 & 2,75 \\
$\mathrm{R}_{\text {Bragg }}$ - fluorita & 2,11 & --- \\
Rwp & 13,60 & 12,40 \\
\hline
\end{tabular}

Para a amostra da mistura CC80F20, os valores dos indicativos $\mathrm{R}_{\mathrm{Bragg}}$ de todas as fases são considerados muito bons, atestando, assim, que o refinamento foi satisfatório. A amostra CC-1500 (sem a adição de fluorita) apresenta ótimos resultados dos indicativos $\mathrm{R}_{\mathrm{Bragg}}$ e bem semelhantes ao da mistura CC80F20, o que indica que o refinamento 


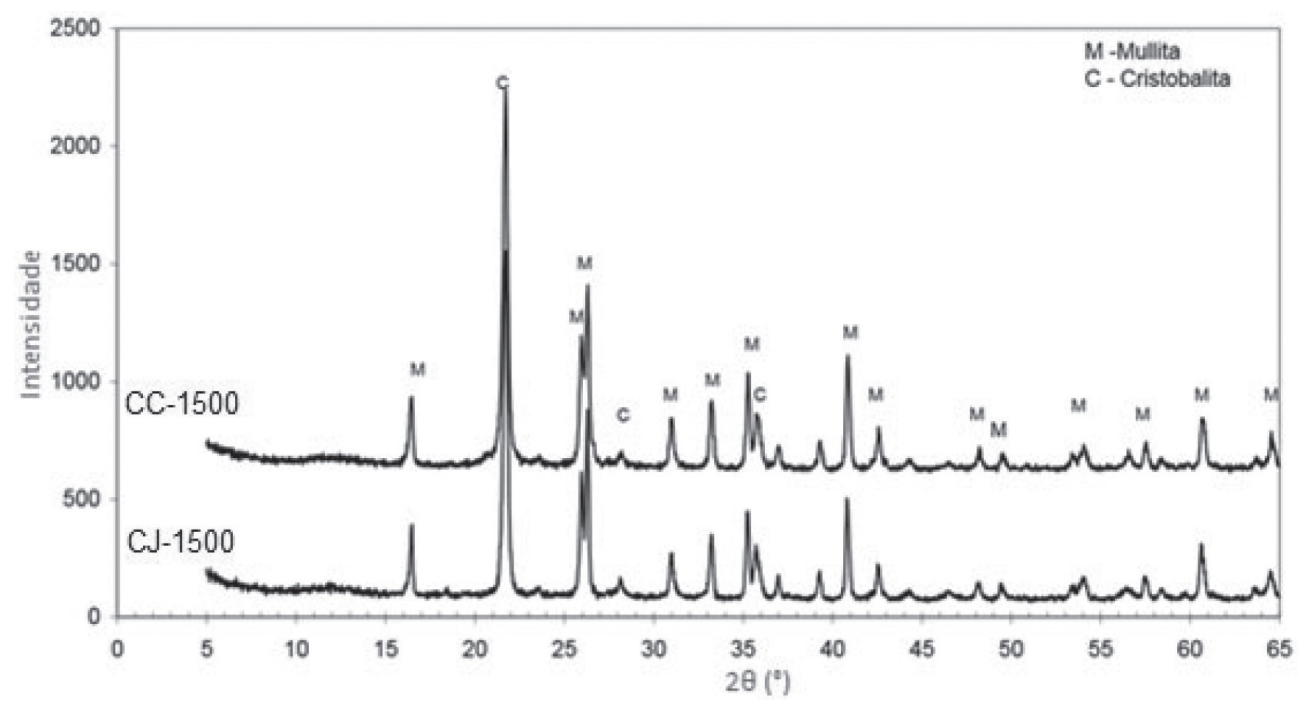

Figura 4. Comparação dos difratogramas de raios X dos rejeitos de caulim CJ e CC calcinados a $1500^{\circ} \mathrm{C}$ por 3 horas no patamar de queima

foi adequado. Os valores de Rwp das duas amostras estão dentro da faixa aceitável (10\% a 20\%), segundo a literatura.

$\mathrm{Na}$ Tabela 5 estão contidos os resultados da quantificação de cada fase presente na amostra CC80F20. Através do resultado do refinamento da amostra com a mistura do padrão interno (CC80F20), pode-se calcular o valor da fase vítrea e utilizá-lo na quantificação das fases da amostra CC-1500. Esse cálculo foi feito pela quantidade de fluorita encontrada pelo refinamento da amostra CC80F20 (21,17\%) e determinação do fator de normalização em relação a quantidade de fluorita $(20,00 \%)$ acrescentada na amostra original.

Tabela 5. Quantificação das fases cristalinas e não cristalina pelo refinamento de Rietveld - Técnica do padrão interno, utilizando o fator de normalização

\begin{tabular}{lccc}
\hline Dados em \% & \multicolumn{3}{c}{ Amostras } \\
\hline $\begin{array}{l}\text { Fases das } \\
\text { Amostras }\end{array}$ & $\begin{array}{c}\text { CC80F20 } \\
\text { Refinamento }\end{array}$ & $\begin{array}{c}\text { CC80F20 } \\
\text { Normalizado } \\
\text { pelo fator }\end{array}$ & $\begin{array}{c}\text { CC80F20 } \\
\text { Cálculo para } \\
\text { 100\% (PR-1500) }\end{array}$ \\
\hline mullita & 52,19 & 49,32 & 61,65 \\
cristobalita & 26,64 & 25,17 & 31,46 \\
fluorita & 21,17 & 20,00 & --- \\
Não Cristalina & --- & 5,51 & 6,89 \\
\hline Total & 100 & 100 & 100 \\
\hline
\end{tabular}

O fator de normalização $\left(f_{n}\right)$ em $100 \%$ CC-1500 foi utilizado para a determinação das quantidades de fases cristalinas e da não cristalina. Os valores percentuais encontrados de cada fase, bem como os teóricos máximos do diagrama de fases $\mathrm{SiO}_{2} / \mathrm{Al}_{2} \mathrm{O}_{3}$ são apresentados na Tabela 6 .

Tabela 6. Quantificação das fases cristalinas e não cristalina nas amostras CC-1500 e CC80F20 e os valores teóricos obtidos do diagrama $\mathrm{SiO}_{2} / \mathrm{Al}_{2} \mathrm{O}_{3}$

\begin{tabular}{lcccc}
\hline Dados em \% & \multicolumn{4}{c}{ Amostras } \\
\hline $\begin{array}{l}\text { Fases das } \\
\text { Amostras }\end{array}$ & $\begin{array}{c}\text { CC-1500 } \\
\text { Refinamento }\end{array}$ & $\begin{array}{c}\text { CC-1500 } \\
\text { Normalizado } \\
\text { pelo fator }\end{array}$ & $\begin{array}{c}\text { CC20F20 } \\
\text { Calo para } \\
100 \% \\
(\mathrm{CC}-1500)\end{array}$ & $\begin{array}{c}\text { Diagrama } \\
\mathrm{SiO}_{2} / \mathrm{Al}_{2} \mathrm{O}_{3}\end{array}$ \\
\hline mullita & 65,93 & 62,30 & 61,65 & 64,00 \\
cristobalita & 34,07 & 32,19 & 31,46 & 36,00 \\
Não Cristalina & --- & 5,51 & 6,89 & --- \\
\hline Total & 100 & 100 & 100 & 100 \\
\hline
\end{tabular}

Conforme as Tabelas 5 e 6 , os valores das fases mullita e cristobalita, para ambas as amostras (a original CC-1500 e a mistura sintética $\mathrm{CC} 80 \mathrm{~F} 20$ ), estão muito próximos um do outro, indicando que o modelo do refinamento aplicado foi ótimo e está bem adequado, pois a amostra de rejeito é a mesma, a diferença está na adição de fluorita em uma delas. Essas fases estão apresentando valores coerentes em comparação com os dados teóricos do diagrama de fases.

A fração em peso (\%) de fase não cristalina na mistura sintética CC80F20, $(5,51 \%)$ foi convertida para a fração em peso (\%) na amostra original CC-1500 resultando no valor real determinado de fase não cristalina de 6,89\%, Equação 13 .

A Tabela 7 mostra os fatores estatísticos de qualidade do refinamento; Rwp, $\mathrm{R}_{\mathrm{e}}$ e GOF determinados pelo programa.

Tabela 7. Fatores de qualidade para as amostras CC $80 \mathrm{~F} 20$ e CC-1500

\begin{tabular}{ccc}
\hline Fatores de qualidade & CC80F20 & CC-1500 \\
\hline Rwp & 13,60 & 12,40 \\
$\mathrm{R}_{\mathrm{e}}$ & 10,80 & 5,42 \\
$\mathrm{GOF}$ & 1,259 & 2,28 \\
\hline
\end{tabular}

Os resultados de $\mathrm{R}_{\mathrm{Bragg}}$, (Tabela 4) são considerados muito bons; os de Rwp estão na faixa típica de refinamento utilizando a difração de raios X e, apesar de os valores de GOF estarem um pouco mais elevados que a unidade, é um valor bem aceitável, visto que ocorreram a presença de fase não cristalina e fases minoritárias.

Os resultados dos fatores de qualidade numéricos podem indicar parcialmente a qualidade do ajuste, porém, também deve-se averiguar se o ajuste está ótimo pela análise visual dos perfis entre os difratogramas, mostrando se o perfil proposto pelo modelo utilizado se adequa ao perfil do difratograma experimental. Pelas Figuras 5 e 6, observam-se graficamente as características das diferenças entre o padrão calculado e o observado nas amostras CC80F20 e CC-1500, respectivamente, mostrando que o refinamento está satisfatório para ambas, analisados também pela linha da diferença entre os dados experimentais $\left(\mathrm{y}_{\mathrm{obs}}\right)$ e os do ajuste do refinamento Rietveld, ( $\left.\mathrm{y}_{\text {calc }}\right)$.

\section{Modelos estruturais}

Partindo dos resultados obtidos pelo programa FullProf, utilizando o parâmetro de rede "a", para estrutura de mullita, nas 


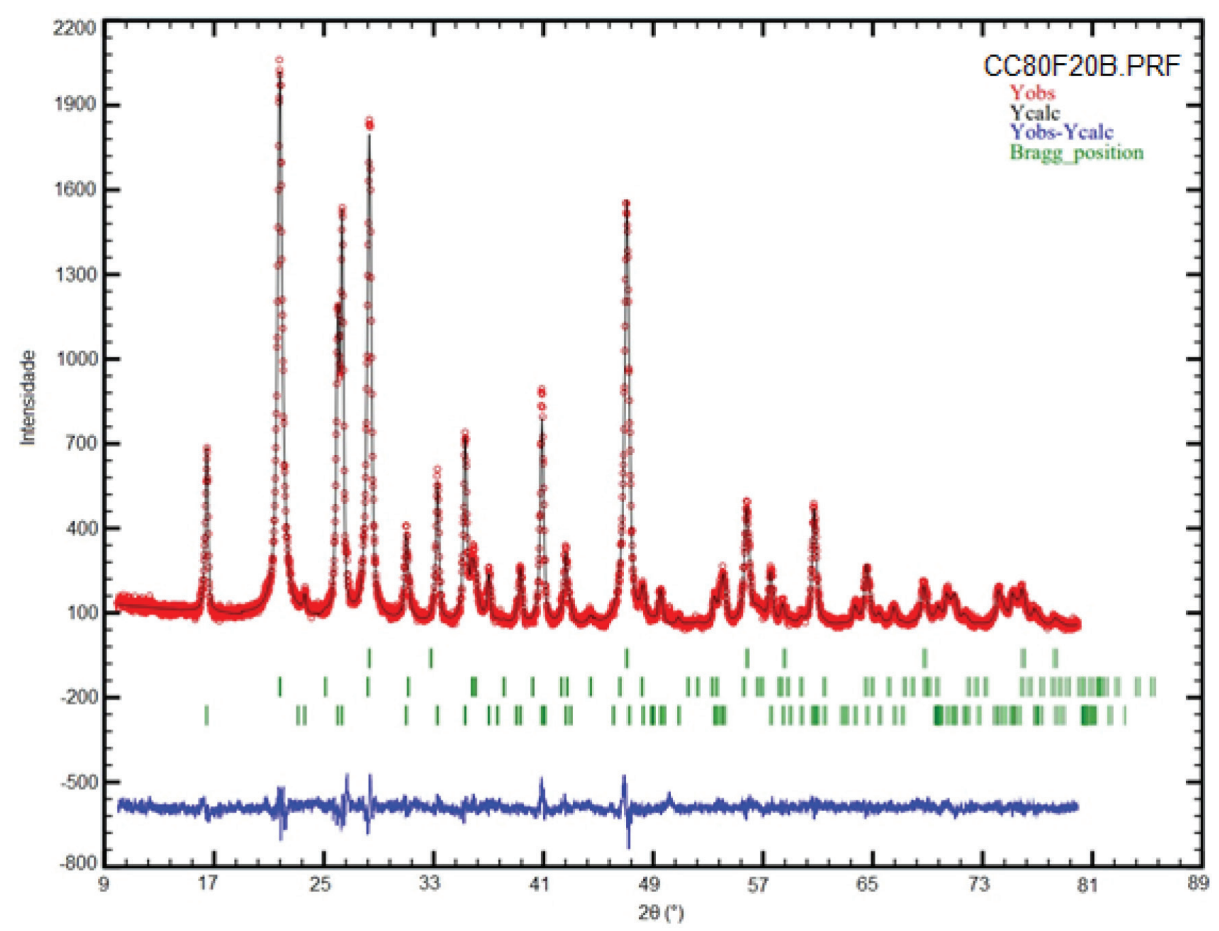

Figura 5. Diferenças entre os difratogramas, calculado e observado da amostra CC80F20

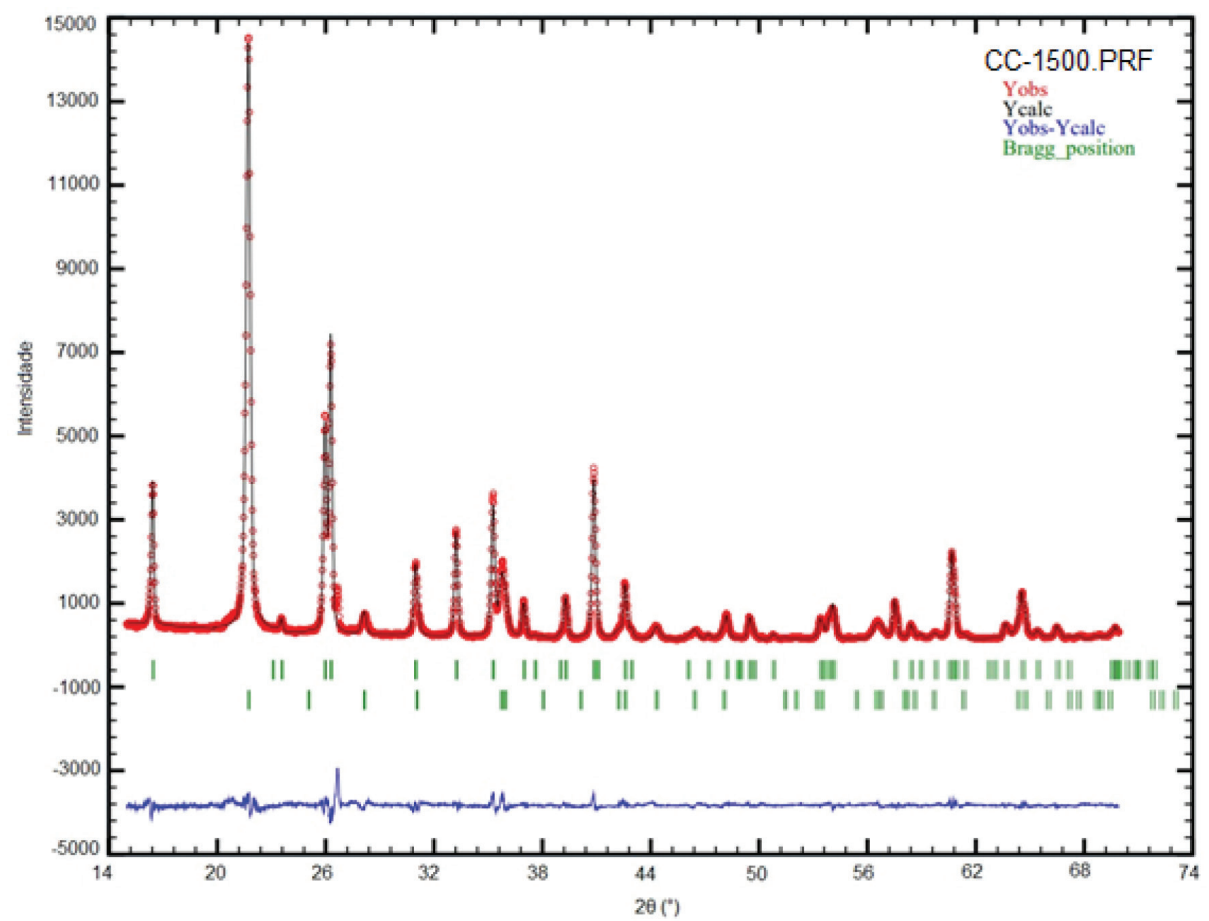

Figura 6. Diferenças entre os difratogramas, calculado e observado da amostra CC-1500

Equações 14 e 15, calcularam-se os valores de "m" e "x", dispostos na Tabela 8.

Pelos resultados obtidos da amostra, a estrutura estudada se aproxima da proporção 3:2 de mullita.

Tabela 8. Parâmetros da estrutura de mullita

\begin{tabular}{cc}
\hline Parâmetro & Valor \\
\hline $\mathrm{a}$ & 7,543408 \\
$\mathrm{~m}$ & 60,52246 \\
$\mathrm{x}$ & 0,262205 \\
\hline
\end{tabular}

Microscopia Eletrônica de Varredura (MEV)

A Figura 7 (a e b) apresenta as imagens no MEV das amostras CJ e CC calcinadas a $1500{ }^{\circ} \mathrm{C}$, preparadas em seção polida. Comparandoas, elas apresentam, em destaque, o interior de um poro com partículas de mullita entrelaçadas, apresentando a morfologia de agulhas bem formadas. Na amostra CJ, as partículas se apresentam maiores e bem mais definidas do que na amostra $\mathrm{CC}$, podendo ser justificado pelo efeito do teor de Fe (cerca de 1,5\%) dentro da estrutura de CJ, como citado anteriormente. 


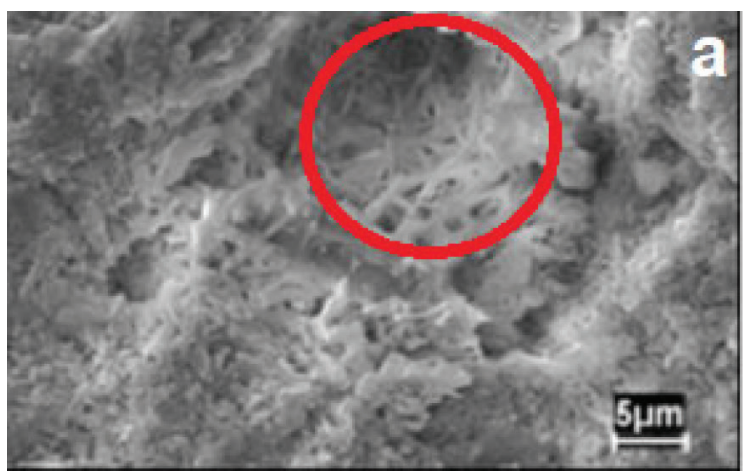

Figura 7. MEV das amostras CJ-1500 (a) e CC-1500 (b), respectivamente

\section{CONCLUSÕES}

Com a aplicação do método de Rietveld, foram quantificadas as fases cristalinas nas amostras de resíduos utilizadas neste trabalho, pois, envolveram-se vários parâmetros de ajustes que influenciaram na quantificação com o uso de todo o perfil difratométrico nos cálculos, superando a questão de sobreposição de picos dos diversos compostos, que é uma das limitações de outros métodos.

As dificuldades encontradas na utilização do método de Rietveld estão: na escolha dos melhores modelos estruturais que representem as fases da amostra, sendo que todas as fases cristalinas devem ser conhecidas; no caso da mullita, por ser uma fase não estequiométrica e, apresentando vacâncias na estrutura, tornando-se assim difícil o refinamento das posições atômicas e dos fatores de ocupação. As imagens das micrografias mostraram que há a presença da morfologia característica da mullita nas duas amostras analisadas de CJ e CC calcinadas a $1500{ }^{\circ} \mathrm{C}$.

Os resultados da quantificação de fases dos dois rejeitos, CC e CJ, calcinados na temperatura de $1500{ }^{\circ} \mathrm{C}$, apresentaram valores bem próximos entre si, sendo identificadas as fases cristalinas por difração de raios X, como de mullita em torno de $66 \%$ e cristobalita por volta de $34 \%$. Esses resultados também são bastante satisfatórios em comparação ao do diagrama de fases teórico, visto que nessas amostras não foram quantificadas as fases não cristalinas.

Pelos resultados da quantificação das fases cristalinas nas amostras CC-1500 e CC80F20, os valores aproximados das fases mullita $(62 \%)$, cristobalita $(32 \%)$ para ambas as amostras estão muito próximos um do outro. Essas fases estão apresentando valores coerentes e muito satisfatórios em comparação com os dados teóricos do diagrama de fases e, pelo método do padrão interno, foi possível determinar a quantificação da fase não cristalina na amostra CC-1500 de aproximadamente $7 \%$.

Pelos indicativos estatísticos dos fatores de qualidade e pelos gráficos dos perfis observados e calculados, pode-se considerar que o modelo do refinamento aplicado está satisfatório, tendo em vista que ocorreram a presença de fase não cristalina e fases minoritárias.

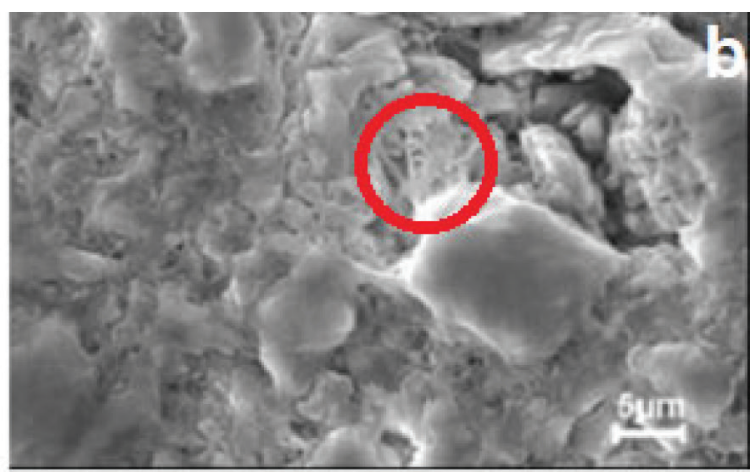

\section{REFERÊNCIAS}

1. Paz, S. P. A.; Angélica, R. S.; Neves, R. F.; Quim. Nova 2010, 33, 579.

2. Carneiro, B. S.; Angélica, R. S.; Scheller, T.; Castro, E. A. S.; Neves, R. F.; Ceramica 2003, 49, 237.

3. Maia, A. A. B.; Saldanha, E.; Angélica, R. S.; Souza, C. A. G.; Neves, R. F.; Ceramica 2007, 53, 319.

4. Martelli, M. C.; Neves, R. F.; Mater. Sci. Forum 2012, 727-728, 697.

5. Schneider, H.; Okada, K.; Pask, J. A.; Mullite and Mullite Ceramics, $1^{\text {st }}$ ed., John Wiley and Sons: Chichester, 1994.

6. Bish, D. L.; Howard, S. A.; J. Appl. Crystallogr. 1988, $21,86$.

7. Rietveld, H. M.; Acta Crystallogr. 1967, 22, 151.

8. Rietveld, H. M.; J. Appl. Crystallogr. 1969, 2, 65.

9. Hill, J. R.; Howard, J. C.; J. Appl. Crystallogr. 1987, 20, 467.

10. De La Torre, A. G.; Bruque, S.; Aranda, A. G.; J. Appl. Crystallogr. 2001, 34, 196.

11. Aramaki, S.; Roy, R.; J. Am. Ceram. Soc. 1962, 45, 229.

12. Aksay, I. A.; Pask, J. A.; J. Am. Ceram. Soc. 1962, 58, 507.

13. Ring, T. A.; Fundamentals of Ceramic Powder Processing and Synthesis, $1^{\text {st }}$ ed., Academic Press: San Diego, 1996, Ch. 16, pp. 781.

14. Young, R. A.; The Rietveld method, Oxford University Press: Oxford, 1993.

15. Pascoal, C.; Machado, R.; Pandolfelli, V. C.; Ceramica 2002, 48, 61.

16. Gonçalves, G. E.; Sabioni, A. C. S.; Ferraz, W. B.; da Costa G. M.; Brito, W.; Dias, J. A.; Garcia, F. A. da C.; REM, Rev. Esc. Minas. 2009, $62,367$.

17. Takei, T.; Kameshima, Y.; Yasumori, A.; Okada, K.; J. Eur. Ceram. Soc. 2001, 21, 2487

18. Monteiro, R. R.; Sabioni, A. C. S.; da Costa, G. M.; Ceramica 2004, 50, 318.

19. Grofcsik, J.; Tamas, F.; Mullite, Its Structure, Formation and Significance, $1^{\text {st }}$ ed., Akademia Kiadó: Budapest, 1961.

20. Araújo, S. M. O.; Dissertação de Mestrado, Universidade Federal do Pará, Brasil, 1995.

21. Melo, V. F.; Schaefer, C. E. G. R.; Singh, B.; Novais, R. F.; Fontes, M. P. F.; Rev. Bras. Cienc. Solo. 2002, 26, 53.

\section{MATERIAL SUPLEMENTAR}

Os arquivos obtidos pelo refinamento da amostra, através da utilização do programa FullProf, estão disponíveis em http:// quimicanova.sbq.org.br, na forma de arquivo PDF, com acesso livre. 